\title{
Certain Admissible Classes of Multivalent Functions
}

\author{
M. K. Aouf, ${ }^{1}$ H. M. Srivastava, ${ }^{2}$ and T. M. Seoudy ${ }^{3,4}$ \\ ${ }^{1}$ Department of Mathematics, Faculty of Science, Mansoura University, Mansoura 35516, Egypt \\ ${ }^{2}$ Department of Mathematics and Statistics, University of Victoria, Victoria, BC, Canada V8W 3R4 \\ ${ }^{3}$ Department of Mathematics, Faculty of Science, Fayoum University, Fayoum 63514, Egypt \\ ${ }^{4}$ The University College at Al-Jamoom, Umm Al-Qura University, Makkah, Saudi Arabia
}

Correspondence should be addressed to T. M. Seoudy; tms00@fayoum.edu.eg

Received 18 July 2014; Accepted 1 September 2014; Published 16 September 2014

Academic Editor: Arcadii Z. Grinshpan

Copyright (C) 2014 M. K. Aouf et al. This is an open access article distributed under the Creative Commons Attribution License, which permits unrestricted use, distribution, and reproduction in any medium, provided the original work is properly cited.

We investigate some applications of the differential subordination and the differential superordination of certain admissible classes of multivalent functions in the open unit disk $\mathbb{U}$. Several differential sandwich-type results are also obtained.

\section{Introduction}

Let $\mathscr{H}(\mathbb{U})$ be the class of functions analytic in the open unit disk

$$
\mathbb{U}=\{z: z \in \mathbb{C},|z|<1\} \text {. }
$$

Denote by $\mathscr{H}[a, n]$ the subclass of $\mathscr{H}(\mathbb{U})$ consisting of functions of the form

$$
f(z)=a+a_{n} z^{n}+a_{n+1} z^{n+1}+\cdots
$$

with

$$
\mathscr{H}=\mathscr{H}[1,1] .
$$

Also let $\mathscr{A}(p)$ be the class of all analytic and $p$-valent functions of the form

$$
f(z)=z^{p}+\sum_{n=p+1}^{\infty} a_{n} z^{n} \quad(p \in \mathbb{N}=\{1,2,3, \ldots\} ; z \in \mathbb{U}) .
$$

Let $f$ and $F$ be members of the function class $\mathscr{H}(\mathbb{U})$. The function $f(z)$ is said to be subordinate to $F(z)$, or the function $F(z)$ is said to be superordinate to $f(z)$, if there exists a function $\omega(z)$, analytic in $\mathbb{U}$ with

$$
\omega(0)=0, \quad|\omega(z)|<1 \quad(z \in \mathbb{U}),
$$

such that

$$
f(z)=F(\omega(z)) .
$$

In such a case we write $f(z) \prec F(z)$. If $F$ is univalent in $\mathbb{U}$, then $f(z) \prec F(z)$ if and only if $f(0)=F(0)$ and $f(\mathbb{U}) \subset$ $F(\mathbb{U})$ (see [1-3]; see also several recent works [4-8] dealing with various properties and applications of the principle of differential subordination and the principle of differential superordination).

We denote by $\mathscr{F}$ the set of all functions $q$ that are analytic and injective on $\overline{\mathbb{U}} \backslash E(q)$, where

$$
E(q)=\left\{\zeta \in \partial \mathbb{U}: \lim _{z \rightarrow \zeta} q(z)=\infty\right\},
$$

and are such that

$$
q^{\prime}(\zeta) \neq 0 \quad(\zeta \in \partial \mathbb{U} \backslash E(q)) .
$$

We further let the subclass of $\mathscr{F}$ for which $q(0)=a$ be denoted by $\mathscr{F}(a)$ and write

$$
\mathscr{F}(1) \equiv \mathscr{F}_{1} \text {. }
$$

In order to prove our results, we will make use of the following classes of admissible functions.

Definition 1 (see [2, p. 27, Definition 2.3a]). Let $\Omega$ be a set in $\mathbb{C}, q \in \mathscr{F}$, and $n \in \mathbb{N}$. The class $\Psi_{n}[\Omega, q]$ of admissible 
functions consists of those functions $\psi: \mathbb{C}^{3} \times \mathbb{U} \rightarrow \mathbb{C}$ that satisfy the following admissibility condition:

$$
\psi(r, s, t ; z) \notin \Omega
$$

whenever

$$
\begin{gathered}
r=q(\zeta), \quad s=k \zeta q^{\prime}(\zeta), \\
\Re\left(\frac{t}{s}+1\right) \geqq k \Re\left(1+\frac{\zeta q^{\prime}(\zeta)}{q^{\prime}(\zeta)}\right),
\end{gathered}
$$

where $z \in \mathbb{U}, \zeta \in \partial \mathbb{U} \backslash E(q)$, and $k \geqq n$. We write $\Psi_{1}[\Omega, q]$ simply as $\Psi[\Omega, q]$.

In particular, if

$$
q(z)=\left(\frac{M z+a}{M+\bar{a} z}\right) M \quad(M>0 ;|a|<M),
$$

then

$$
q(\mathbb{U})=\mathbb{U}_{M}=\{w:|w|<M\},
$$

$q(0)=a, E(q)=\emptyset$, and $q \in \mathscr{F}(a)$. In this case, we set $\Psi_{n}[\Omega, M, a]=\Psi_{n}[\Omega, q]$. Moreover, in the special case, when we set $\Omega=\mathbb{U}_{M}$, the class is simply denoted by $\Psi_{n}[M, a]$.

Definition 2 (see [3, p. 817, Definition 3]). Let $\Omega$ be a set in $\mathbb{C}, q \in \mathscr{H}[a, n]$ with $q^{\prime}(z) \neq 0$. The class $\Psi_{n}^{\prime}[\Omega, q]$ of admissible functions consists of those functions $\psi: \mathbb{C}^{3} \times \overline{\mathbb{U}} \rightarrow$ $\mathbb{C}$ that satisfy the following admissibility condition:

$$
\psi(r, s, t ; \zeta) \in \Omega
$$

whenever

$$
\begin{gathered}
r=q(z), \quad s=\frac{z q^{\prime}(z)}{m}, \\
\mathfrak{R}\left(\frac{t}{s}+1\right) \leqq \frac{1}{m} \mathfrak{R}\left(1+\frac{z q^{\prime \prime}(z)}{q^{\prime}(z)}\right),
\end{gathered}
$$

where $z \in \mathbb{U}, \zeta \in \partial \mathbb{U}$, and $m \geqq n \geqq 1$. In particular, we write $\Psi_{1}^{\prime}[\Omega, q]$ simply as $\Psi^{\prime}[\Omega, q]$.

In our investigation we need the following lemmas which are proved by Miller and Mocanu (see [2] and [3]).

Lemma 3 (see [2, p. 28, Theorem 2.3b]). Let $\psi \in \Psi_{n}[\Omega, q]$ with $q(0)=a$. If the analytic function $g(z)$ given by

$$
g(z)=a+a_{n} z^{n}+a_{n+1} z^{n+1}+\cdots
$$

satisfies the inclusion relationship

$$
\psi\left(g(z), z g^{\prime}(z), z^{2} g^{\prime \prime}(z) ; z\right) \in \Omega,
$$

then $g \prec q$.

Lemma 4 (see [3, p. 818, Theorem 1]). Let $\psi \in \Psi_{n}^{\prime}[\Omega, q]$ with $q(0)=a$. If $g \in \mathscr{F}(a)$ and the function

$$
\psi\left(g(z), z g^{\prime}(z), z^{2} g^{\prime \prime}(z) ; z\right)
$$

is univalent in $\mathbb{U}$, then

$$
\Omega \subset\left\{\psi\left(g(z), z g^{\prime}(z), z^{2} g^{\prime \prime}(z) ; z\right): z \in \mathbb{U}\right\}
$$

implies that $q \prec g$.
In this paper, we determine the sufficient conditions for certain admissible classes of multivalent functions so that

$$
q_{1}(z) \prec\left(\frac{f(z)}{z^{p}}\right)^{\mu} \prec q_{2}(z),
$$

where $\mu>0$ and $q_{1}$ and $q_{2}$ are given univalent functions in $\mathbb{U}$ with

$$
q_{1}(0)=q_{2}(0)=1
$$

In addition, we derive several differential sandwich-type results. A similar problem for analytic functions involving certain operators was studied by Aghalary et al. [9], Ali et al. [10], Aouf et al. [11], Kim and Srivastava [12], and other authors (see [13-15]). In particular, unlike the earlier investigation by Aouf and Seoudy [16], we have not used any operators in our present investigation. Nevertheless, for the benefit of the targeted readers of our paper, in addition to oft-cited paper [11], we have included several further citations of recent works (see, e.g., [17-21]) in which various families of linear operators were applied in conjunction with the principle of differential subordination and the principle of differential superordination for the study of analytic or meromorphic multivalent functions.

\section{A Set of Subordination Results}

Unless otherwise mentioned, we assume throughout this paper that $p \in \mathbb{N}, \mu>0, z \in \mathbb{U}$, and all power functions are tacitly assumed to denote their principal values.

Definition 5. Let $\Omega$ be a set in $\mathbb{C}$ and $q \in \mathscr{F}_{1} \cap \mathscr{H}$. The class $\Phi[\Omega, q, p, \mu]$ of admissible functions consists of those functions $\phi: \mathbb{C}^{3} \times \mathbb{U} \rightarrow \mathbb{C}$ that satisfy the following admissibility condition:

$$
\phi(u, v, w ; z) \notin \Omega
$$

whenever

$$
\begin{gathered}
u=q(\zeta), \quad v=\frac{k \zeta q^{\prime}(\zeta)+\mu p q(\zeta)}{\mu p}, \\
\Re\left(\frac{w-(2 \mu p-1) v+\mu p u}{v-u}\right) \geqq k \mathfrak{R}\left(1+\frac{\zeta q^{\prime \prime}(\zeta)}{q^{\prime}(\zeta)}\right),
\end{gathered}
$$

where $z \in \mathbb{U}, \zeta \in \partial \mathbb{U} \backslash E(q)$, and $k \geqq 1$. For simplicity, we write

$$
\Phi[\Omega, q, p, 1]=\Phi[\Omega, q, p] .
$$

Theorem 6. Let $\phi \in \Phi[\Omega, q, p, \mu]$. If $f \in \mathscr{A}(p)$ satisfies the condition

$$
\begin{aligned}
& \left\{\phi \left(\left(\frac{f(z)}{z^{p}}\right)^{\mu},\left(\frac{f(z)}{z^{p}}\right)^{\mu} \frac{z f^{\prime}(z)}{p f(z)},\left(\frac{f(z)}{z^{p}}\right)^{\mu} \frac{z^{2} f^{\prime \prime}(z)}{p f(z)}\right.\right. \\
& \left.\left.\quad+(\mu-1) p\left(\frac{f(z)}{z^{p}}\right)^{\mu}\left(\frac{z f^{\prime}(z)}{p f(z)}\right)^{2} ; z\right): z \in \mathbb{U}\right\} \subset \Omega,
\end{aligned}
$$


then

$$
\left(\frac{f(z)}{z^{p}}\right)^{\mu} \prec q(z) .
$$

Proof. We begin by defining the analytic function $g$ in $\mathbb{U}$ by

$$
g(z)=\left(\frac{f(z)}{z^{p}}\right)^{\mu} \quad(z \in \mathbb{U}) .
$$

Then, in view of (27), we get

$$
\left(\frac{f(z)}{z^{p}}\right)^{\mu} \frac{z f^{\prime}(z)}{p f(z)}=\frac{z g^{\prime}(z)+\mu p g(z)}{\mu p} .
$$

Further computations show that

$$
\begin{gathered}
\left(\frac{f(z)}{z^{p}}\right)^{\mu} \frac{z^{2} f^{\prime \prime}(z)}{p f(z)}+(\mu-1) p\left(\frac{f(z)}{z^{p}}\right)^{\mu}\left(\frac{z f^{\prime}(z)}{p f(z)}\right)^{2} \\
=\frac{z^{2} g^{\prime \prime}(z)+2 \mu p z g^{\prime}(z)+\mu p(\mu p-1) g(z)}{\mu p} .
\end{gathered}
$$

We now define the transformations from $\mathbb{C}^{3}$ to $\mathbb{C}$ by

$$
\begin{gathered}
u=r, \quad v=\frac{s+\mu p r}{\mu p}, \\
w=\frac{t+2 \mu p s+\mu p(\mu p-1) r}{\mu p}
\end{gathered}
$$

and suppose that

$$
\begin{aligned}
\psi(r, s, t ; z) & =\phi(u, v, w ; z) \\
& =\phi\left(r, \frac{s+\mu p r}{\mu p}, \frac{t+2 \mu p s+\mu p(\mu p-1) r}{\mu p} ; z\right) .
\end{aligned}
$$

The proof will make use of Lemma 3. Indeed, by using (27) to (31), we obtain

$$
\begin{aligned}
& \psi\left(g(z), z g^{\prime}(z), z^{2} g^{\prime \prime}(z) ; z\right) \\
& =\phi\left(\left(\frac{f(z)}{z^{p}}\right)^{\mu},\left(\frac{f(z)}{z^{p}}\right)^{\mu} \frac{z f^{\prime}(z)}{p f(z)},\right. \\
& \quad\left(\frac{f(z)}{z^{p}}\right)^{\mu} \frac{z^{2} f^{\prime \prime}(z)}{p f(z)} \\
& \left.\quad+(\mu-1) p\left(\frac{f(z)}{z^{p}}\right)^{\mu}\left(\frac{z f^{\prime}(z)}{p f(z)}\right)^{2} ; z\right) .
\end{aligned}
$$

Hence (25) becomes

$$
\psi\left(g(z), z g^{\prime}(z), z^{2} g^{\prime \prime}(z) ; z\right) \in \Omega .
$$

The proof is completed if it can be shown that the admissibility condition for $\phi \in \Phi[\Omega, q, p, \mu]$ is equivalent to the admissibility condition for $\psi$ as given in Definition 1 . We note that

$$
\frac{t}{s}+1=\frac{w-(2 \mu p-1) v+\mu p u}{v-u},
$$

and hence $\psi \in \Psi_{1}[\Omega, q]$. By Lemma 3 , we thus obtain

$$
g(z) \prec q(z) \quad \text { or } \quad\left(\frac{f(z)}{z^{p}}\right)^{\mu} \prec q(z) .
$$

which evidently proves Theorem 6 .

If $\Omega \neq \mathbb{C}$ is a simply connected domain, then $\Omega=$ $h(\mathbb{U})$ for some conformal mapping $h$ of $\mathbb{U}$ onto $\Omega$. In this case, the class $\Phi[h(\mathbb{U}), q, p, \mu]$ is written, for convenience, as $\Phi[h, q, p, \mu]$. The following result is an immediate consequence of Theorem 6 .

Theorem 7. Let $\phi \in \Phi[h, q, p, \mu]$. If $f \in \mathscr{A}(p)$ satisfies the condition,

$$
\begin{gathered}
\phi\left(\left(\frac{f(z)}{z^{p}}\right)^{\mu},\left(\frac{f(z)}{z^{p}}\right)^{\mu} \frac{z f^{\prime}(z)}{p f(z)},\left(\frac{f(z)}{z^{p}}\right)^{\mu} \frac{z^{2} f^{\prime \prime}(z)}{p f(z)}\right. \\
\left.+(\mu-1) p\left(\frac{f(z)}{z^{p}}\right)^{\mu}\left(\frac{z f^{\prime}(z)}{p f(z)}\right)^{2} ; z\right) \prec h(z),
\end{gathered}
$$

then

$$
\left(\frac{f(z)}{z^{p}}\right)^{\mu} \prec q(z) .
$$

Putting $\mu=1$ in Theorem 7, we obtain the following corollary.

Corollary 8. Let $\phi \in \Phi[h, q, p]$. If $f \in \mathscr{A}(p)$ satisfies the condition

$$
\phi\left(\frac{f(z)}{z^{p}}, \frac{f^{\prime}(z)}{p z^{p-1}}, \frac{f^{\prime \prime}(z)}{p z^{p-2}}\right) \prec h(z)
$$

then

$$
\frac{f(z)}{z^{p}} \prec q(z) .
$$

Our next result is an extension of Theorem 6 to the case where the behavior of $q$ on $\partial \mathbb{U}$ is not known.

Corollary 9. Let $\Omega \subset \mathbb{C}$ and suppose that the function $q$ is univalent in $\mathbb{U}$ with $q(0)=1$. Also let $\phi \in \Phi\left[\Omega, q_{\rho}, p, \mu\right]$ for some $\rho \in(0,1)$, where $q_{\rho}(z)=q(\rho z)$. If $f \in \mathscr{A}(p)$ and

$$
\begin{gathered}
\phi\left(\left(\frac{f(z)}{z^{p}}\right)^{\mu},\left(\frac{f(z)}{z^{p}}\right)^{\mu} \frac{z f^{\prime}(z)}{p f(z)},\left(\frac{f(z)}{z^{p}}\right)^{\mu} \frac{z^{2} f^{\prime \prime}(z)}{p f(z)}\right. \\
\left.+(\mu-1) p\left(\frac{f(z)}{z^{p}}\right)^{\mu}\left(\frac{z f^{\prime}(z)}{p f(z)}\right)^{2} ; z\right) \in \Omega,
\end{gathered}
$$

then

$$
\left(\frac{f(z)}{z^{p}}\right)^{\mu} \prec q(z) .
$$


Proof. Theorem 6 readily yields

$$
\left(\frac{f(z)}{z^{p}}\right)^{\mu} \prec q_{\rho}(z)
$$

The asserted result is now deduced from the fact that $q_{\rho}(z) \prec$ $q(z)$.

Theorem 10. Let the functions $h$ and $q$ be univalent in $\mathbb{U}$, with $q(0)=1$, and set

$$
q_{\rho}(z)=q(\rho z), \quad h_{\rho}(z)=h(\rho z) .
$$

Also let $\phi: \mathbb{C}^{3} \times \mathbb{U} \rightarrow \mathbb{C}$ satisfy one of the following conditions:

(1) $\phi \in \Phi\left[h, q_{\rho}, p, \mu\right]$ for some $\rho \in(0,1)$ or

(2) there exists $\rho_{0} \in(0,1)$ such that $\phi \in \Phi\left[h_{\rho}, q_{\rho}, p, \mu\right]$ for all $\rho \in\left(\rho_{0}, 1\right)$.

If $f \in \mathscr{A}(p)$ satisfies condition (36), then

$$
\left(\frac{f(z)}{z^{p}}\right)^{\mu} \prec q(z)
$$

Proof. The proof of Theorem 10 is similar to the proof of a known result $[2$, p. 30 , Theorem $2.3 \mathrm{~d}]$ and is, therefore, omitted.

The next theorem yields the best dominant of differential subordination (36).

Theorem 11. Let the function $h$ be univalent in $\mathbb{U}$. Also let $\phi$ : $\mathbb{C}^{3} \times \mathbb{U} \rightarrow \mathbb{C}$. Suppose that the differential equation

$$
\phi\left(q(z), z q^{\prime}(z), z^{2} q^{\prime \prime}(z) ; z\right)=h(z)
$$

has a solution $q$ with $q(0)=1$ and satisfies one of the following conditions:

(1) $q \in \mathscr{F}_{1}$ and $\phi \in \Phi[h, q, p, \mu]$;

(2) the function $q$ is univalent in $\mathbb{U}$ and $\phi \in \Phi\left[h, q_{\rho}, p, \mu\right]$ for some $\rho \in(0,1)$; or

(3) the function $q$ is univalent in $\mathbb{U}$ and there exists $\rho_{0} \in$ $(0,1)$ such that $\phi \in \Phi\left[h_{\rho}, q_{\rho}, p, \mu\right]$ for all $\rho \in\left(\rho_{0}, 1\right)$. If $f \in \mathscr{A}(p)$ satisfies (36), then

$$
\left(\frac{f(z)}{z^{p}}\right)^{\mu} \prec q(z)
$$

and $q$ is the best dominant.

Proof. Following the same arguments in [2, p. 31, Theorem 2.3e], we deduce that $q$ is a dominant from Theorems 7 and 10. Since $q$ satisfies (45), it is also a solution of (36) and, therefore, $q$ will be dominated by all dominants. Hence $q$ is the best dominant.

In the particular case when $q(z)=1+M z(M>0)$, in view of Definition 5 , the class $\Phi[\Omega, q, p, \mu]$ of admissible functions, denoted by $\Phi[\Omega, M, p, \mu]$, is described below.
Definition 12. Let $\Omega$ be a set in $\mathbb{C}$ and $M>0$. The class $\Phi[\Omega, M, p, \mu]$ of admissible functions consists of those functions $\phi: \mathbb{C}^{3} \times \mathbb{U} \rightarrow \mathbb{C}$ such that

$$
\begin{aligned}
& \phi\left(1+M e^{i \theta}, 1+\frac{k+\mu p}{\mu p} M e^{i \theta},\right. \\
& \left.\quad \frac{L+\mu p\left[(2 k+\mu p-1) M e^{i \theta}+\mu p-1\right]}{\mu p} ; z\right) \notin \Omega
\end{aligned}
$$

whenever $z \in \mathbb{U}, \theta \in \mathbb{R}$, and

$$
\Re\left(L e^{-i \theta}\right) \geqq(k-1) k M
$$

for all real $\theta$ and $k \geqq \mu p$.

Corollary 13. Let $\phi \in \Phi[\Omega, M, p, \mu]$. If $f \in \mathscr{A}(p)$ satisfies the condition

$$
\begin{gathered}
\phi\left(\left(\frac{f(z)}{z^{p}}\right)^{\mu},\left(\frac{f(z)}{z^{p}}\right)^{\mu} \frac{z f^{\prime}(z)}{p f(z)},\left(\frac{f(z)}{z^{p}}\right)^{\mu} \frac{z^{2} f^{\prime \prime}(z)}{p f(z)}\right. \\
\left.+(\mu-1) p\left(\frac{f(z)}{z^{p}}\right)^{\mu}\left(\frac{z f^{\prime}(z)}{p f(z)}\right)^{2} ; z\right) \in \Omega,
\end{gathered}
$$

then

$$
\left|\left(\frac{f(z)}{z^{p}}\right)^{\mu}-1\right|<M \quad(z \in \mathbb{U}) .
$$

In the special case when

$$
\Omega=q(\mathbb{U})=\{\omega:|\omega-1|<M\},
$$

the class $\Phi[\Omega, M, p, \mu]$ is simply denoted by $\Phi[M, p, \mu]$.

Corollary 14. Let $\phi \in \Phi[M]$. If $f \in \mathscr{A}(p)$ satisfies the condition

$$
\begin{aligned}
& \mid \phi\left(\left(\frac{f(z)}{z^{p}}\right)^{\mu},\left(\frac{f(z)}{z^{p}}\right)^{\mu} \frac{z f^{\prime}(z)}{p f(z)},\left(\frac{f(z)}{z^{p}}\right)^{\mu} \frac{z^{2} f^{\prime \prime}(z)}{p f(z)}\right. \\
& \left.+(\mu-1) p\left(\frac{f(z)}{z^{p}}\right)^{\mu}\left(\frac{z f^{\prime}(z)}{p f(z)}\right)^{2} ; z\right)-1 \mid<M,
\end{aligned}
$$

then

$$
\left|\left(\frac{f(z)}{z^{p}}\right)^{\mu}-1\right|<M .
$$

Corollary 15. If $k \geqq 1$ and $f \in \mathscr{A}(p)$ satisfies the condition

$$
\left|\left(\frac{f(z)}{z^{p}}\right)^{\mu} \frac{z f^{\prime}(z)}{p f(z)}-1\right|<M,
$$

then

$$
\left|\left(\frac{f(z)}{z^{p}}\right)^{\mu}-1\right|<M .
$$

Proof. Corollary 15 follows from Corollary 14 upon setting

$$
\phi(u, v, w ; z)=v=1+\left(\frac{k+\mu p}{\mu p}\right) M e^{i \theta} .
$$




\section{Superordination and Sandwich-Type Results}

In this section we investigate the dual problem of differential subordination, that is, differential superordination of multivalent functions. For this purpose, the class of admissible functions is given in the following definition.

Definition 16. Let $\Omega$ be a set in $\mathbb{C}$ and $q \in \mathscr{H}$ with $z q^{\prime}(z) \neq$ 0 . The class $\Phi^{\prime}[\Omega, q, p, \mu]$ of admissible functions consists of those functions $\phi: \mathbb{C}^{3} \times \overline{\mathbb{U}} \rightarrow \mathbb{C}$ that satisfy the following admissibility condition:

$$
\phi(u, v, w ; \zeta) \in \Omega
$$

whenever

$$
\begin{gathered}
u=q(z), \quad v=\frac{z q^{\prime}(z)+m \mu p q(z)}{m \mu p}, \\
\Re\left(\frac{w-(2 \mu p-1) v+\mu p u}{v-u}\right) \geqq \frac{1}{m} \mathfrak{R}\left(1+\frac{\zeta q^{\prime \prime}(\zeta)}{q^{\prime}(\zeta)}\right),
\end{gathered}
$$

where $z \in \mathbb{U}, \zeta \in \partial \mathbb{U}$, and $m \geqq 1$. For convenience, we write

$$
\Phi^{\prime}[\Omega, q, p, 1]=\Phi^{\prime}[\Omega, q, p] .
$$

Theorem 17. Let $\phi \in \Phi^{\prime}[\Omega, q, p, \mu]$. If $f \in \mathscr{A}(p)$,

$$
\begin{gathered}
\left(\frac{f(z)}{z^{p}}\right)^{\mu} \in \mathscr{F}_{1} \\
\phi\left(\left(\frac{f(z)}{z^{p}}\right)^{\mu},\left(\frac{f(z)}{z^{p}}\right)^{\mu} \frac{z f^{\prime}(z)}{p f(z)},\left(\frac{f(z)}{z^{p}}\right)^{\mu} \frac{z^{2} f^{\prime \prime}(z)}{p f(z)}\right. \\
\left.+(\mu-1) p\left(\frac{f(z)}{z^{p}}\right)^{\mu}\left(\frac{z f^{\prime}(z)}{p f(z)}\right)^{2} ; z\right)
\end{gathered}
$$

is univalent in $\mathbb{U}$, then

$$
\begin{gathered}
\Omega \subset\left\{\phi \left(\left(\frac{f(z)}{z^{p}}\right)^{\mu},\left(\frac{f(z)}{z^{p}}\right)^{\mu} \frac{z f^{\prime}(z)}{p f(z)},\left(\frac{f(z)}{z^{p}}\right)^{\mu} \frac{z^{2} f^{\prime \prime}(z)}{p f(z)}\right.\right. \\
\left.\left.+(\mu-1) p\left(\frac{f(z)}{z^{p}}\right)^{\mu}\left(\frac{z f^{\prime}(z)}{p f(z)}\right)^{2} ; z\right): z \in \mathbb{U}\right\}
\end{gathered}
$$

implies that

$$
q(z) \prec\left(\frac{f(z)}{z^{p}}\right)^{\mu}
$$

Proof. From (32) and (61), we find that

$$
\Omega \subset\left\{\psi\left(g(z), z g^{\prime}(z), z^{2} g^{\prime \prime}(z) ; z\right): z \in \mathbb{U}\right\} .
$$

We also see from (30) that the admissibility condition for the function class $\phi \in \Phi^{\prime}[\Omega, q, p, \mu]$ is equivalent to the admissibility condition for $\psi$ as given in Definition 2 . Hence $\psi \in \Psi_{1}^{\prime}[\Omega, q]$. Thus, by Lemma 4 , we have

$$
q(z) \prec g(z) \quad \text { or } \quad q(z) \prec\left(\frac{f(z)}{z^{p}}\right)^{\mu},
$$

which evidently completes the proof of Theorem 17.

If $\Omega \neq \mathbb{C}$ is a simply connected domain, then $\Omega=h(\mathbb{U})$ for some conformal mapping $h$ of $\mathbb{U}$ onto $\Omega$. In this case, the class $\Phi^{\prime}[h(\mathbb{U}), q, p, \mu]$ is written simply as $\Phi^{\prime}[h, q, p, \mu]$.

Proceeding similarly as in Section 2, the following result can be derived as an immediate consequence of Theorem 17.

Theorem 18. Let the function $h$ be analytic in $\mathbb{U}$ and $\phi \in$ $\Phi^{\prime}[h, q, p, \mu]$. If $f \in \mathscr{A}(p)$,

$$
\begin{gathered}
\left(\frac{f(z)}{z^{p}}\right)^{\mu} \in \mathscr{F}_{1}, \\
\phi\left(\left(\frac{f(z)}{z^{p}}\right)^{\mu},\left(\frac{f(z)}{z^{p}}\right)^{\mu} \frac{z f^{\prime}(z)}{p f(z)},\left(\frac{f(z)}{z^{p}}\right)^{\mu} \frac{z^{2} f^{\prime \prime}(z)}{p f(z)}\right. \\
\left.+(\mu-1) p\left(\frac{f(z)}{z^{p}}\right)^{\mu}\left(\frac{z f^{\prime}(z)}{p f(z)}\right)^{2} ; z\right)
\end{gathered}
$$

is univalent in $\mathbb{U}$, then

$$
\begin{aligned}
h(z) \prec \phi( & \left(\frac{f(z)}{z^{p}}\right)^{\mu},\left(\frac{f(z)}{z^{p}}\right)^{\mu} \frac{z f^{\prime}(z)}{p f(z)},\left(\frac{f(z)}{z^{p}}\right)^{\mu} \frac{z^{2} f^{\prime \prime}(z)}{p f(z)} \\
& \left.+(\mu-1) p\left(\frac{f(z)}{z^{p}}\right)^{\mu}\left(\frac{z f^{\prime}(z)}{p f(z)}\right)^{2} ; z\right)
\end{aligned}
$$

implies that

$$
q(z) \prec\left(\frac{f(z)}{z^{p}}\right)^{\mu} .
$$

Putting $\mu=1$ in Theorem 18, we obtain the following corollary.

Corollary 19. Let the function $h$ be analytic in $\mathbb{U}$ and $\phi \in$ $\Phi^{\prime}[h, q, p]$. If $f \in \mathscr{A}(p)$,

$$
\begin{gathered}
\frac{f(z)}{z^{p}} \in \mathscr{F}_{1}, \\
\phi\left(\frac{f(z)}{z^{p}}, \frac{f^{\prime}(z)}{p z^{p-1}}, \frac{f^{\prime \prime}(z)}{p z^{p-2}} ; z\right)
\end{gathered}
$$

is univalent in $\mathbb{U}$, then

$$
h(z) \prec \phi\left(\frac{f(z)}{z^{p}}, \frac{f^{\prime}(z)}{p z^{p-1}}, \frac{f^{\prime \prime}(z)}{p z^{p-2}} ; z\right)
$$

implies that

$$
q(z) \prec \frac{f(z)}{z^{p}}
$$


Theorems 17 and 18 can only be used to obtain subordinants of the differential superordination of the form (61) or (66). The following theorem proves the existence of the best subordinant of (66) for a specified $\phi$.

Theorem 20. Let the function $h$ be analytic in $\mathbb{U}$ and $\phi: \mathbb{C}^{3} \times$ $\overline{\mathbb{U}} \rightarrow \mathbb{C}$. Suppose that the differential equation

$$
\phi\left(q(z), z q^{\prime}(z), z^{2} q^{\prime \prime}(z) ; z\right)=h(z)
$$

has a solution $q \in \mathscr{F}_{1}$. If $\phi \in \Phi^{\prime}[h, q, p, \mu], f \in \mathscr{A}(p)$,

$$
\begin{gathered}
\left(\frac{f(z)}{z^{p}}\right)^{\mu} \in \mathscr{F}_{1} \\
\phi\left(\left(\frac{f(z)}{z^{p}}\right)^{\mu},\left(\frac{f(z)}{z^{p}}\right)^{\mu} \frac{z f^{\prime}(z)}{p f(z)},\left(\frac{f(z)}{z^{p}}\right)^{\mu} \frac{z^{2} f^{\prime \prime}(z)}{p f(z)}\right. \\
\left.+(\mu-1) p\left(\frac{f(z)}{z^{p}}\right)^{\mu}\left(\frac{z f^{\prime}(z)}{p f(z)}\right)^{2} ; z\right)
\end{gathered}
$$

is univalent in $\mathbb{U}$, then

$$
\begin{gathered}
h(z) \prec \phi\left(\left(\frac{f(z)}{z^{p}}\right)^{\mu},\left(\frac{f(z)}{z^{p}}\right)^{\mu} \frac{z f^{\prime}(z)}{p f(z)},\left(\frac{f(z)}{z^{p}}\right)^{\mu} \frac{z^{2} f^{\prime \prime}(z)}{p f(z)}\right. \\
\left.+(\mu-1) p\left(\frac{f(z)}{z^{p}}\right)^{\mu}\left(\frac{z f^{\prime}(z)}{p f(z)}\right)^{2} ; z\right)
\end{gathered}
$$

implies that

$$
q(z) \prec\left(\frac{f(z)}{z^{p}}\right)^{\mu}
$$

and $q$ is the best subordinant.

Proof. The proof is similar to the proof of Theorem 11. We, therefore, omit the details involved.

Combining Theorems 7 and 18, we obtain the following sandwich-type theorem.

Theorem 21. Let the functions $h_{1}$ and $q_{1}$ be analytic in $\mathbb{U}$, the function $h_{2}$ univalent in $\mathbb{U}, q_{2} \in \mathscr{F}_{1}$ with

$$
\begin{gathered}
q_{1}(0)=q_{2}(0)=1, \\
\phi \in \Phi\left[h_{2}, q_{2}, p, \mu\right] \cap \Phi^{\prime}\left[h_{1}, q_{1}, p, \mu\right] .
\end{gathered}
$$

If $f \in \mathscr{A}(p)$,

$$
\begin{gathered}
\left(\frac{f(z)}{z^{p}}\right)^{\mu} \in \mathscr{H} \cap \mathscr{F}_{1}, \\
\phi\left(\left(\frac{f(z)}{z^{p}}\right)^{\mu},\left(\frac{f(z)}{z^{p}}\right)^{\mu} \frac{z f^{\prime}(z)}{p f(z)},\left(\frac{f(z)}{z^{p}}\right)^{\mu} \frac{z^{2} f^{\prime \prime}(z)}{p f(z)}\right. \\
\left.+(\mu-1) p\left(\frac{f(z)}{z^{p}}\right)^{\mu}\left(\frac{z f^{\prime}(z)}{p f(z)}\right)^{2} ; z\right)
\end{gathered}
$$

is univalent in $\mathbb{U}$, then

$$
\begin{aligned}
h_{1}(z) \prec \phi( & \left(\frac{f(z)}{z^{p}}\right)^{\mu},\left(\frac{f(z)}{z^{p}}\right)^{\mu} \frac{z f^{\prime}(z)}{p f(z)},\left(\frac{f(z)}{z^{p}}\right)^{\mu} \frac{z^{2} f^{\prime \prime}(z)}{p f(z)} \\
& \left.+(\mu-1) p\left(\frac{f(z)}{z^{p}}\right)^{\mu}\left(\frac{z f^{\prime}(z)}{p f(z)}\right)^{2} ; z\right) \prec h_{2}(z)
\end{aligned}
$$

implies that

$$
q_{1}(z) \prec\left(\frac{f(z)}{z^{p}}\right)^{\mu} \prec q_{2}(z) .
$$

Upon setting $\mu=1$ in Theorem 21, we get the following result.

Corollary 22. Let the functions $h_{1}$ and $q_{1}$ be analytic in $\mathbb{U}$, the function $h_{2}$ univalent in $\mathbb{U}, q_{2} \in \mathscr{F}_{1}$ with

$$
q_{1}(0)=q_{2}(0)=1,
$$

and $\phi \in \Phi\left[h_{2}, q_{2}, p\right] \cap \Phi^{\prime}\left[h_{1}, q_{1}, p\right]$. If $f \in \mathscr{A}(p)$,

$$
\begin{gathered}
\frac{f(z)}{z^{p}} \in \mathscr{H} \cap \mathscr{F}_{1}, \\
\phi\left(\frac{f(z)}{z^{p}}, \frac{f^{\prime}(z)}{p z^{p-1}}, \frac{f^{\prime \prime}(z)}{p z^{p-2}}\right)
\end{gathered}
$$

is univalent in $\mathbb{U}$, then

$$
h_{1}(z) \prec \phi\left(\frac{f(z)}{z^{p}}, \frac{f^{\prime}(z)}{p z^{p-1}}, \frac{f^{\prime \prime}(z)}{p z^{p-2}}\right) \prec h_{2}(z)
$$

implies that

$$
q_{1}(z) \prec \frac{f(z)}{z^{p}} \prec q_{2}(z) .
$$

\section{Conflict of Interests}

The authors declare that there is no conflict of interests regarding the publication of this paper.

\section{References}

[1] T. Bulboacă, Differential Subordinations and Superordinations: Recent Results, House of Scientific Book Publishers, ClujNapoca, Romania, 2005.

[2] S. S. Miller and P. T. Mocanu, Differential Subordinations : Theory and Applications, vol. 225 of Monographs and Textbooks in Pure and Applied Mathematics, Marcel Dekker, New York, NY, USA, 2000.

[3] S. S. Miller and P. T. Mocanu, "Subordinants of differential superordinations," Complex Variables. Theory and Application, vol. 48, no. 10, pp. 815-826, 2003. 
[4] N. E. Cho, T. Bulboacă, and H. M. Srivastava, "A general family of integral operators and associated subordination and superordination properties of some special analytic function classes," Applied Mathematics and Computation, vol. 219, no. 4, pp. 2278-2288, 2012.

[5] K. Kuroki, H. M. Srivastava, and S. Owa, "Some applications of the principle of differential subordination," Electronic Journal of Mathematical Analysis and Applications, vol. 1, article 5, pp. 4046, 2013.

[6] H. M. Srivastava and A. Y. Lashin, "Subordination properties of certain classes of multivalently analytic functions," Mathematical and Computer Modelling, vol. 52, no. 3-4, pp. 596-602, 2010.

[7] Q.-H. Xu, C.-B. Lv, N.-C. Luo, and H. M. Srivastava, "Sharp coefficient estimates for a certain general class of spirallike functions by means of differential subordination," Filomat, vol. 27, no. 7, pp. 1351-1356, 2013.

[8] Q.-H. Xu, H.-G. Xiao, and H. M. Srivastava, "Some applications of differential subordination and the Dziok-Srivastava convolution operator," Applied Mathematics and Computation, vol. 230, pp. 496-508, 2014.

[9] R. Aghalary, R. M. Ali, S. B. Joshi, and V. Ravichandran, "Inequalities for analytic functions defined by certain linear operators," International Journal of Mathematical Sciences, vol. 4, no. 2, pp. 267-274, 2005.

[10] R. M. Ali, V. Ravichandran, and N. Seenivasagan, "Differential subordination and superordination of analytic functions defined by the multiplier transformation," Mathematical Inequalities \& Applications, vol. 12, no. 1, pp. 123-139, 2009.

[11] M. K. Aouf, H. M. Hossen, and A. Y. Lashin, "An application of certain integral operators," Journal of Mathematical Analysis and Applications, vol. 248, no. 2, pp. 475-481, 2000.

[12] Y. C. Kim and H. M. Srivastava, "Inequalities involving certain families of integral and convolution operators," Mathematical Inequalities \& Applications, vol. 7, no. 2, pp. 227-234, 2004.

[13] M. K. Aouf, "Inequalities involving certain integral operators," Journal of Mathematical Inequalities, vol. 2, no. 4, pp. 537-547, 2008.

[14] M. K. Aouf and T. M. Seoudy, "Differential subordination and superordination of analytic functions defined by an integral operator," European Journal of Pure and Applied Mathematics, vol. 3, no. 1, pp. 26-44, 2010.

[15] M. K. Aouf and T. M. Seoudy, "Differential subordination and superordination of analytic functions defined by certain integral operator," Acta Universitatis Apulensis, no. 24, pp. 211-229, 2010.

[16] M. K. Aouf and T. M. Seoudy, "Subordination and superordination of certain linear operator on meromorphic functions," Annales Universitatis Mariae Curie-Skłodowska A, vol. 64, no. 1, pp. 1-16, 2010.

[17] N. E. Cho, "Sandwich-type theorems for meromorphic multivalent functions associated with the Liu-Srivastava operator," Acta Mathematica Scientia B, vol. 32, no. 3, pp. 929-941, 2012.

[18] N. E. Cho and R. Srivastava, "Subordination properties for a general class of integral operators involving meromorphically multivalent functions," Advances in Difference Equations, vol. 2013, article 93, 15 pages, 2013.

[19] H. E. Darwish, A. Y. Lashin, and B. H. Rizqan, "Sandwich-type theorems for meromorphic multivalent functions associated with a linear operator," International Journal of Scientific \& Engineering Research, vol. 5, pp. 169-185, 2014.
[20] R. W. Ibrahim and M. Darus, "Certain studies of the LiuSrivastava linear operator on meromorphic functions," International Journal of Physical Sciences, vol. 6, no. 15, pp. 3736-3739, 2011.

[21] H. Tang, H. M. Srivastava, S.-H. Li, and L.-N. Ma, “Thirdorder differential subordination and superordination results for meromorphically multivalent functions associated with the Liu-Srivastava operator," Abstract and Applied Analysis, vol. 2014, Article ID 792175, 11 pages, 2014. 


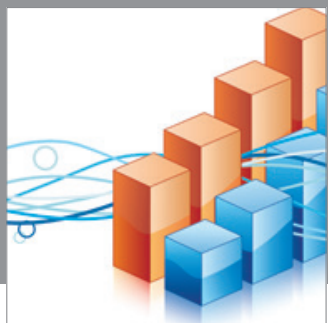

Advances in

Operations Research

mansans

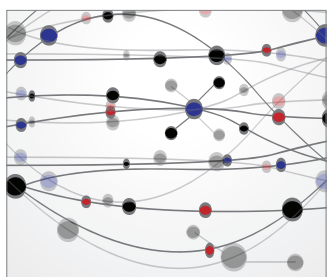

The Scientific World Journal
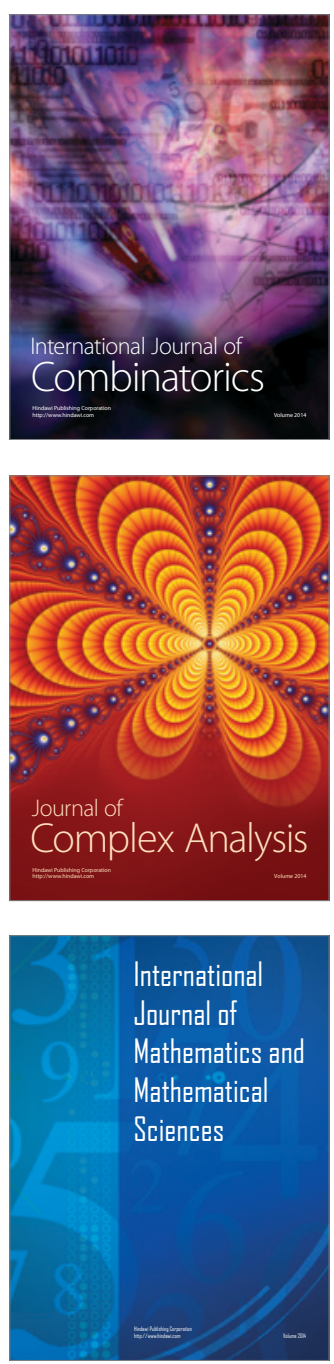
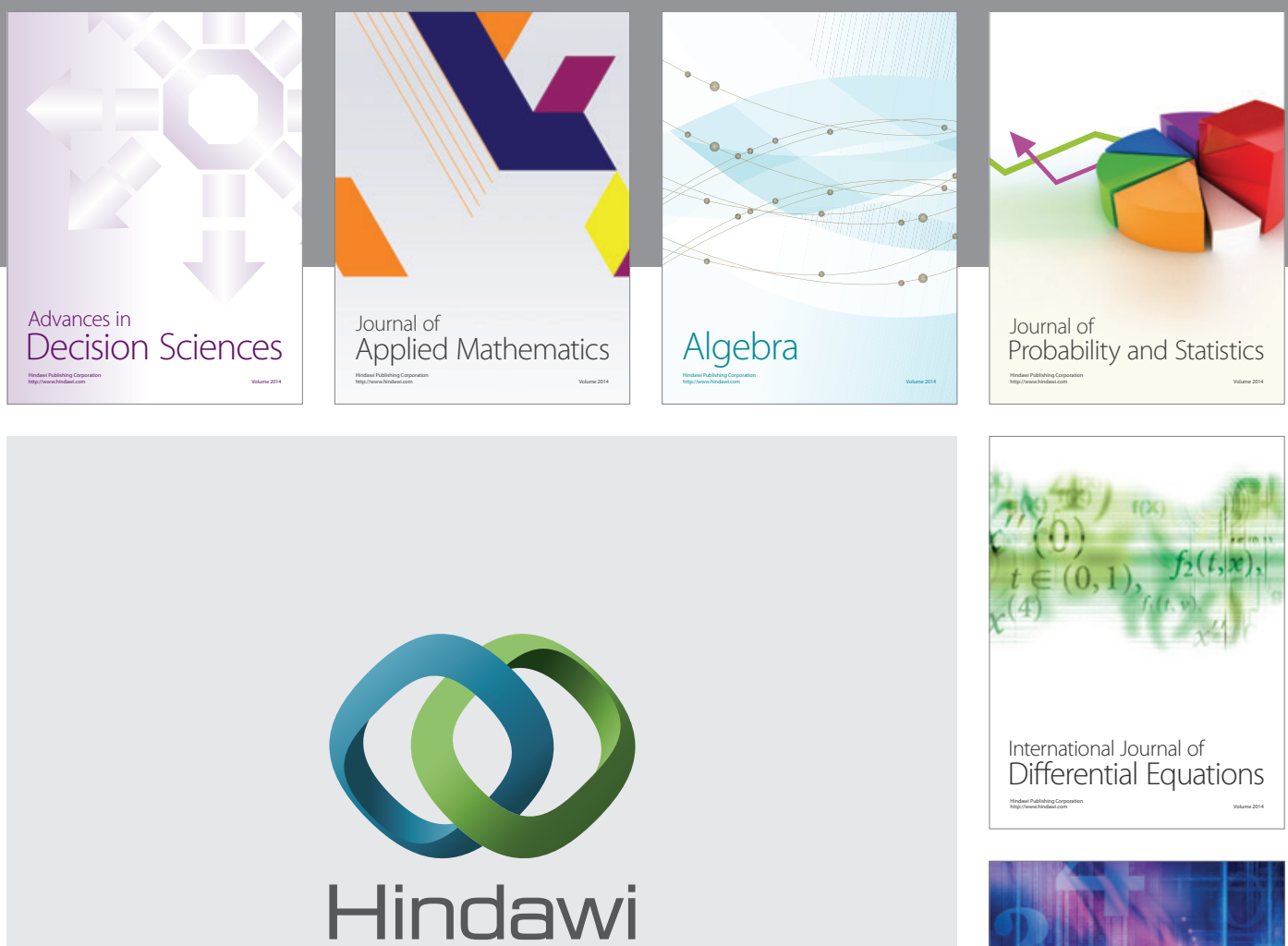

Submit your manuscripts at http://www.hindawi.com
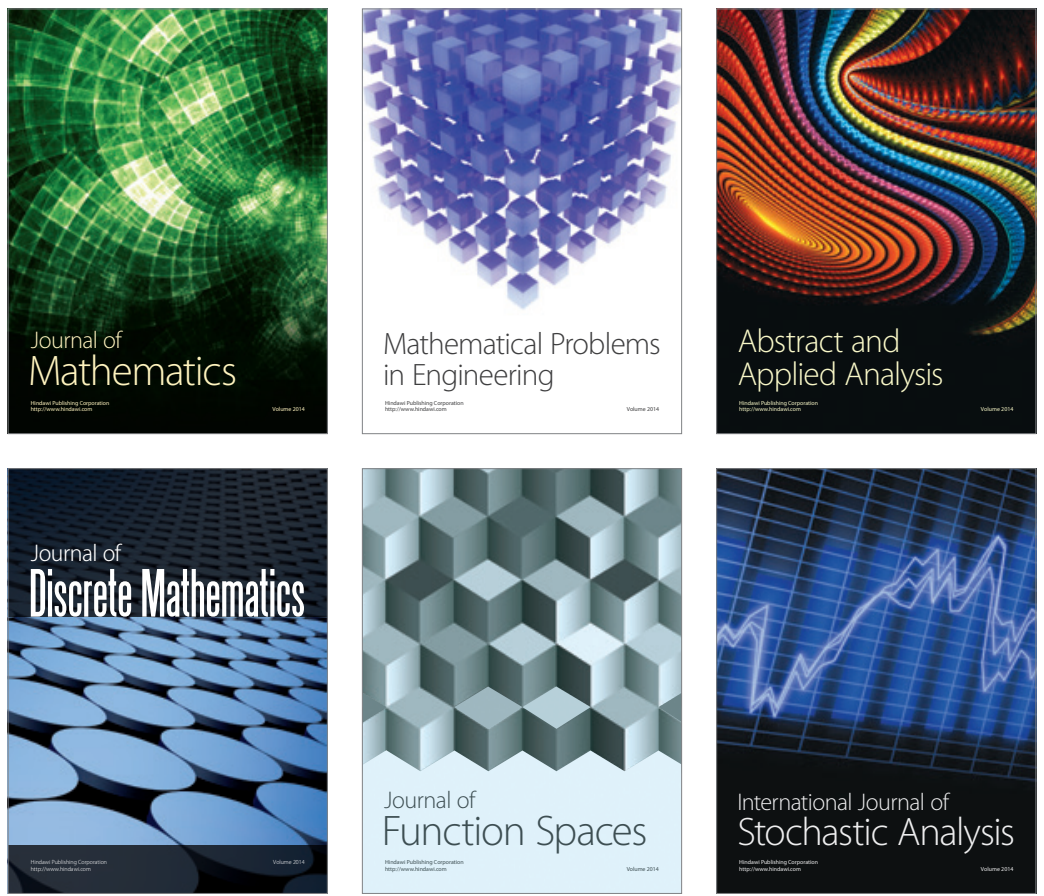

Journal of

Function Spaces

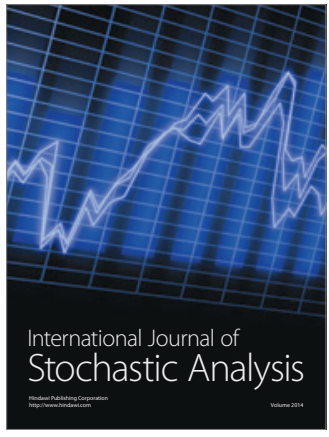

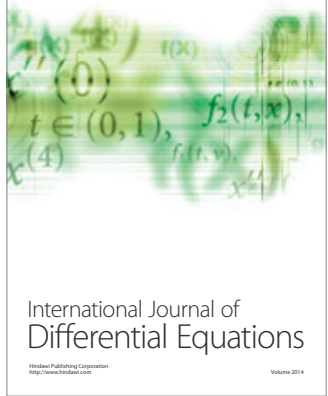
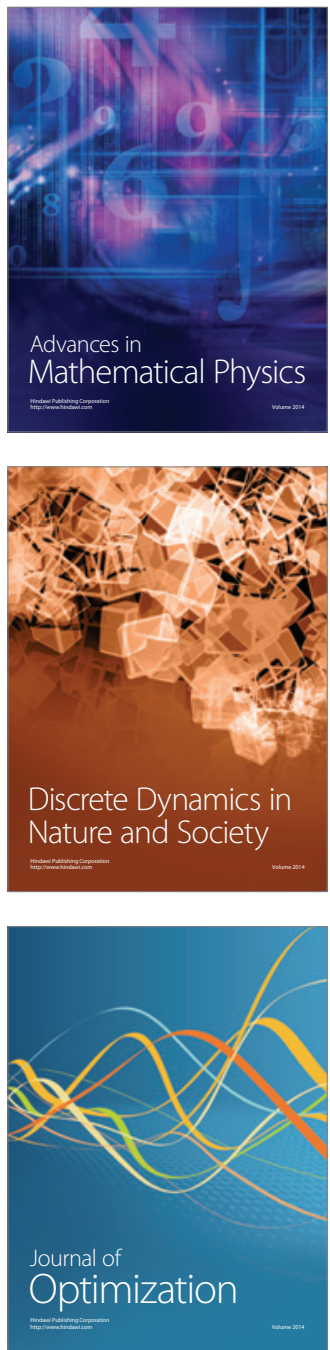